\title{
TEORIA DEI GIOCHI, SCIENZE SOCIALI E NEUROFISIOLOGIA
}

In questo saggio ricostruiremo la storia di una proficua contaminazione tra idee appartenenti non soltanto a discipline diverse, ma addirittura a tre distinti campi disciplinari, quali sono le scienze formali, le scienze sociali e le scienze naturali. Più precisamente, prenderemo in esame in chiave diacronica le sinergie tra i modelli matematici della teoria dei giochi (con particolare attenzione all'ultimatum game), alcuni meccanismi d'interazione umana studiati dalle scienze sociali, in primis dall'economia, e le più recenti scoperte della neurofisiologia. Non è nostra intenzione ricostruire la storia completa della teoria dei giochi, né gli aspetti strettamente tecnici della stessa, quanto piuttosto un particolare percorso all'interno di questa storia, al fine di evidenziare il modo in cui detta teoria ha interagito con le scienze sociali e le neuroscienze. La tesi principale che andiamo a sostenere è che l'approccio bottom-up della game theory, al contrario di quello top-down della rational choice theory, permette di rimanere vicini alla griglia empirica dei comportamenti umani e, nel contempo, di raggiungere un livello esplicativo e predittivo ben superiore a quello che di norma forniscono le nozioni del senso comune. Questo approccio sinergico pone dunque le basi per la creazione di un ponte tra il piano sperimentale e il piano teorico, restando nell'ambito del paradigma dell'individualismo metodologico.

\section{LE ORIGINI DELIA TEORIA DEI GIOCHI}

La teoria dei giochi (game theory) è stata variamente denominata anche "scienza della strategia" (science of strategy) ', "scienza del ragionamento strategico" (science of strategic reasoning), o "teoria delle decisioni interattive" (interactive decision theory) ${ }^{2}$.

1 Dixit/Nalebuff: Game Theory.

2 Aumann: Game theory. 
Sulla Stanford Encyclopedia of Philosophy, Don Ross definisce la teoria dei giochi come "lo studio dei modi in cui le interazioni strategiche tra agenti economici producono risultati in relazione alle preferenze (o utilità) di detti agenti, dove i risultati in questione potrebbero non essere stati voluti da alcuno degli agenti» ${ }^{3}$. Possiamo vedere intanto che l'applicazione della teoria dei giochi viene per definizione limitata agli agenti "economici". La storia dimostra però che la game theory trascende l'ambito delle decisioni economiche ${ }^{4}$, come anche il prosieguo dell'articolo di Ross mostra piuttosto bene. Il che rende forse più adeguata la definizione proposta da Roger Myerson, secondo il quale la teoria dei giochi è "lo studio dei modelli matematici del conflitto e della cooperazione tra decisori intelligenti e razionali»s.

Piuttosto perentoriamente, Ross afferma anche che "la teoria matematica dei giochi è stata inventata da John von Neumann e Oskar Morgenstern (1944) ”. Questa è in effetti una tesi comunemente accettata e non senza un fondamento. Va però precisato che il libro Theory of Games and Economic Behavior ${ }^{7}$ di Neumann e Morgenstern rappresenta soltanto il momento in cui detta teoria diventa popolare negli Stati Uniti d'America, mentre i suoi inizi vanno ritrovati più indietro nel tempo, in Europa ${ }^{8}$.

Come per tutto ciò che è filosoficamente significativo, per rintracciare l'origine di questa idea, si potrebbe risalire ai Greci e, precisamente, ai tempi delle Guerre del Peloponneso, nel V secolo A.C. È ciò che fa lo stesso Ross, quando spiega che già in due dialoghi platonici, il Lachete e il Simposio, Socrate analizza la Battaglia di Delio tra Ateniesi e Tebani secondo uno schema di strategie e decisioni interattive che può essere ricondotto proprio alla teoria dei giochi.

Paul Walker, che ha recentemente compilato una cronologia (pur incompleta) della teoria dei giochi, concorda sul fatto che i secoli a cui bisogna guardare sono i cinque che precedono la nostra era. Tuttavia, egli guarda ad Oriente. Un modello di ragionamento strategico è stato infatti individuato in un codice di leggi vigente nell'antica Babilonia: il Talmud babilonese.

Uno dei problemi discussi nel Talmud è il cosiddetto problema del contratto matrimonialc: un uomo ha tre mogli il cui contratto matrimoniale specifica

3 Ross: Game Theory.

4 Per fare solo un esempio, nel 2013, Wojciech Załuski ha pubblicato un libro sulle applicazioni della teoria dei giochi nel campo del diritto: Game Theory in Jurisprudence.

5 Myerson: Game Theory. Analysis of Conflict, p. 1.

6 Ross: Game Theory.

7 Neumann/Morgenstern: Theory of Games and Economic Behavior.

8 La voce "Game Theory" del Dictionary of the History of Ideas (pp. 263-275) riconduce gli inizi di questa scienza agli studi di Cardano, Laplace, Bernoulli, Leibniz ed altri, anche se non manca di sottolineare che è stato il libro di Neumann e Morgenstern che "ha definitivamente stabilito il campo». Si tratta di una ricostruzione storica particolarmente importante, considerando che a firmarla è Oskar Morgenstern in persona. 
che in caso di sua morte riceveranno rispettivamente 100, 200 e 300. Il Talmud fornisce raccomandazioni apparentemente contraddittorie. Se l'uomo muore lasciando un patrimonio di soli 100 , il Talmud raccomanda la divisione in parti uguali. Tuttavia, se il patrimonio vale 300 , esso raccomanda la suddivisione proporzionale $(50,100,150)$, mentre per un patrimonio pari a 200 , la sua raccomandazione di $(50,75,75)$ è un mistero vero e proprio. Questo particolare Mishna ha sconcertato gli studiosi del Talmud per duemila anni. Nel 1985 è stato stabilito che il Talmud anticipa la moderna teoria dei giochi cooperativi. Ogni soluzione corrisponde al nucleolo di un gioco appropriatamente definito?

Il matematico John Nash sottolinea che anche Machiavelli analizza come "giochi" le lotte per il potere nelle corti del Rinascimento, in particolare dello Stato Pontificio, e come giocatori gli attori politici che vi prendono parte. Dunque, in questi scritti politologici, emergono le premesse essenziali per la successiva nascita della teoria dei giochi. Lo stesso studioso spiega però che la versione formalizzata o matematica della teoria muove i primi passi quasi due secoli più tardi, e precisamente in uno scambio epistolare tra Blaise Pascal e Pierre de Fermat, due tra i matematici più affermati del XVII secolo. Le lettere che si scambiavano i due luminari vertevano essenzialmente sulle probabilità nelle situazioni di gioco, in particolare nei giochi d'azzardo praticati nei casinò. La capacità di memorizzare le informazioni disponibili, per esempio, è correlata positivamente con la probabilità di vincere a certi giochi ad informazione parziale ${ }^{10}$.

Un'altra tappa dello sviluppo della teoria dei giochi è il libro di Augustin Cournot Researches into the Mathematical Principles of the Theory of Wealth, apparso nel 1838. Nel capitolo 7, intitolato "On the Competition of Producers", l'autore discute il caso speciale del duopolio, fornendo una spiegazione intuitiva di quello che sarà poi formalizzato come l'equilibrio di Nash. Cournot fornisce anche una nozione dinamica o evolutiva di risposta ottimale all'azione degli altri.

Nel 1881, si registra la pubblicazione del libro Mathematical Psychics: An Essay on the Application of Mathematics to the Moral Sciences, di Francis Ysidro Edgeworth. Lautore propone la curva dei contratti come una soluzione del problema di determinare il risultato dello scambio commerciale tra individui. "In un mondo in cui esistono due beni e due tipi di consumatori, egli ha dimostrato che la curva dei contratti si restringe all'insieme degli equilibri competitivi quando il numero dei consumatori di ogni tipo diventa infinito ${ }^{11}$. L'importanza di questo studio è dovuta al fatto che il concetto di "nucleo" (in inglese: core) della teoria dei giochi è proprio una generalizzazione della curva dei contratti di Edgeworth.

Il primo teorema formale della teoria dei giochi viene proposto nel $1913 \mathrm{dal}$ matematico tedesco Ernst Zermelo e applicato al gioco degli scacchi. Al teorema

9 Walker: A Chronology of Game Theory.

10 Nash: La teoria dei giochi.

11 Walker: A Chronology of Game Theory. 
e ai suoi sviluppi è stato dedicato uno studio da parte di Ulrich Schwalbe e Paul Walker ${ }^{12}$, in appendice del quale è inclusa anche una traduzione dell'articolo originale di Zermelo "On an Application of Set Theory to the Theory of the Game of Chess"'13.

Sebbene questi studi possono essere fatti a buon diritto rientrare nella game theory, l'espressione "teoria dei giochi" - come lo stesso Nash chiarisce - compare soltanto negli anni Venti del XX secolo, ancora una volta in Francia. È infatti il matematico francese Émile Borel a coniare l'espressione "théorie des jeux", per denominare una serie di quattro studi da lui effettuati. L'Encyclopaedia Britannica conferma infatti che Borel "ha completato una serie di articoli sulla teoria dei giochi (1921-1927) ed è diventato il primo a definire i giochi di strategian" ${ }^{14}$. Borel si occupava di giochi a somma zero con due giocatori. E cercò di trovare un buon concetto di "soluzione". In particolare, viene rimarcato che in Algebre et calcul des probabilites" 15 il matematico francese "fornisce il primo indizio che la randomizzazione può garantire un risultato stabile nell'ambito delle strategie miste» ${ }^{16}$.

Nonostante tutti gli studi finora citati abbiano una certa importanza, è tuttavia comunemente accettato che il contributo davvero significativo per la nascita della teoria dei giochi, come campo di studio autonomo e scientificamente rilevante, va individuato nel lavoro del matematico tedesco John von Neumann. I "giochi di strategia" sono infatti stati formalizzati e matematizzati da Neumann, in alcuni lavori pubblicati tra le due guerre mondiali. Il primo articolo appare nel $1928 \mathrm{e}$ porta il titolo "Zur Theorie der Gesellschaftsspiele"17.

Sedici anni più tardi, nel 1944, compare il lavoro chiave di questo campo di studi, ovvero il già citato Theory of Games and Economic Behavior, scritto a quattro mani da von Neumann e dall'economista Oskar Morgenstern. Limportanza di questo libro non risiede tanto nel fatto che si tratta della prima pubblicazione in inglese sull'argomento (questo è rilevante per il mondo anglosassone, ma non particolarmente significativo per l'Europa continentale), quanto piuttosto nel fatto che vi si trova la prima applicazione di questo strumento matematico alle scienze sociali. Il testo analizza infatti giochi cooperativi di diversi giocatori, soprattutto nell'ambito delle relazioni economiche, ma fa intuire anche la possibilità dell'applicazione della game theory su vasta scala, per affrontare problemi non solo inerenti le strategie economiche, ma anche politiche e militari. Di qui, l'idea di includere questa teoria matematica nel programma di svariati corsi di laurea. Per questo pre-

12 Schwalbe/Walker: Zermelo and the Early History of Game Theory.

13 Zermelo: Über eine Anwendung der Mengenlehre auf die Theorie des Schachspiels.

14 [Anonymus]: Émile Borel.

15 Borel: Algèbre et calcul des probabilités.

16 Hossain/Niyato/Han: Dynamic Spectrum Access and Management in Cognitive Radio Networks, p. 156.

17 Neumann: Zur Theorie der Gesellschaftsspiele. 
ciso motivo, possiamo convenire che il lavoro di Neumann e Morgenstern segna la nascita della teoria dei giochi moderna.

Che la teoria dei giochi sia rilevante per le scienze sociali nel loro complesso, e non solo per l'economia, è stato sottolineato da diversi studiosi. Per esempio, nel 1983, l'antropologo Cliffort Geertz ha affermato quanto segue:

L'analogia dei giochi è sempre più popolare nella teoria sociale contemporanea e necessita sempre più di un esame critico. L'impulso a vedere l'uno o l'altro tipo di comportamento sociale come l'uno o l'altro tipo di gioco proviene da un certo numero di fonti. Le più importanti sono la concezione delle forme di vita come giochi linguistici di Wittgenstein, la visione ludica della cultura di Huizinga, e la nuova scienza della strategia elaborata da von Neumann e Morgenstern in Theory of Games and Economic Behavior ${ }^{18}$.

Geertz menziona insieme la teoria dei giochi e i cosiddetti "game studies" (espressione mantenuta nell'originale inglese anche nei testi italiani, o talvolta resa con il termine "ludologia"). Si deve notare che i campi della game theory e dei game studies, pur avendo il termine "game" in comune, sono piuttosto distanti tra loro. Si sono infatti sviluppati in modo indipendente, con scopi e metodi differenti, anche se è vero che entrambi gli approcci hanno influenzato le scienze sociali e di conseguenza hanno interagito all'interno di quest'area disciplinare. Questo è potuto accadere, perché i due campi di studio sono in linea di principio interdisciplinari e, dunque, strutturalmente predisposti alle contaminazioni.

È tuttavia importante chiarire che, in questo articolo, concentreremo l'attenzione sulla sola teoria dei giochi. La regola del pollice proposta da Richard Swedberg per distinguere le diverse fonti di influenza è la seguente: quando si fa riferimento a "ricompense", "strategie" e "matrici", allora stiamo parlando di teoria dei giochi in senso stretto e non dei word-games di Ludwig Wittgenstein o dei game studies di Johan Huizinga.

\section{LA TEORIA DEI GIOCHI NEL SECONDO DOPOGUERRA}

Come qualsiasi altra teoria scientifica, la teoria dei giochi ha dunque molti padri. Robert K. Merton ci ha mostrato come quasi tutte le idee scientifiche siano in realtà "scoperte multiple", ovvero effettuate indipendentemente da diversi ricercatori in luoghi diversi ${ }^{19}$. Le scoperte e le innovazioni sono nell'aria, dipendono da un certo clima culturale e da un certo stato di avanzamento della ricerca. È per- 
ciò poco sorprendente che ricercatori intelligenti e preparati, in diversi paesi, arrivino alle stesse conclusioni. Purtroppo, questa verità "sociologica" è ancora troppo spesso misconosciuta, nonostante la mole di dati empirici a suo sostegno. Gli scienziati preferiscono attribuire la scoperta interamente al proprio genio, piuttosto che riconoscere l'importanza del fattore ambientale o il semplice fatto che non possono restringere a se stessi l'insieme delle persone dotate di un fine intelletto. Perciò, non di rado, essi rifutano di dividere il merito con altri ricercatori e si lanciano in spiacevoli lotte per la priorità che rivelano i lati umani peggiori.

Lo stesso Merton ha pure più volte sottolineato che una scienza che esita a dimenticare i suoi fondatori, ovvero che resta ferma alle opere dei fondatori, è perduta. Questo non è accaduto alla game theory, la quale ha fatto progressi molto grandi nella seconda metà del XX secolo. Basti pensare che il gioco più conosciuto e popolare della teoria delle decisioni interattive non era ancora presente nell'opera seminale di Neumann e Morgenstern.

$\mathrm{Ci}$ riferiamo naturalmente al "dilemma del prigioniero", che viene elaborato nel 1950 da Melvin Dresher e Merrill Flood della Rand Corporation e poi perfezionato da Albert W. Tucker ${ }^{20}$. Si tratta di un canonico esempio di gioco che mostra la dinamica complessa del conflitto e della cooperazione tra due agenti razionali. Più precisamente, questo gioco mostra che - in particolari circostanze - due individui potrebbero non cooperare, anche se sarebbe nel loro interesse farlo. Così Dario Fürst riassume il gioco:

Due uomini sono incolpati di aver commesso insieme un grave delitto, ma il pubblico ministero sa che non vi sono prove sufficienti per condannarli. Egli li esorta (separatamente) a confessare: se uno solo confessa, avrà una pena particolarmente lieve come premio per la sua collaborazione, mentre l'altro avrà quella massima; se confessano entrambi avranno una pena inferiore alla massima, ma tuttavia severa; se nessuno dei due confessa potranno essere condannati per un reato minore (per esempio possesso illegale di armi) e la pena sarà ovviamente leggera, però maggiore di quella che costituirebbe il "premio di collaborazione" prima menzionato. Un esempio concreto è dato dalla seguente matrice, dove la strategia 1 significa per entrambi i giocatori 'non confessare', quella 2 'confessare' e i risultati sono anni di detenzione. Traducendo questi risultati in unità convenzionali di utilità (fatta variare tra $0 \mathrm{e} 1$ ) si ottiene una matrice del tipo seguente.

\begin{tabular}{c|cc} 
I & \multicolumn{2}{|c}{ II } \\
\hline 1 & 1 & 2 \\
2 & $\begin{array}{c}(0,9 ; 0,9) \\
(1 ; 0)\end{array}$ & $(0 ; 1)$ \\
& (0,1) 0,1$)$
\end{tabular}

Matrice 6. 
Nell'ipotesi non cooperativa, cioè se i due prigionieri non possono comunicare tra loro, si vede subito che per ciascuno dei due la strategia 2 domina, in senso stretto, la 1 (fissata la strategia di uno dei due, il massimo dell'utilità dell'altro corrisponde alla strategia 2). Dunque, avendo entrambi lo scopo di massimizzare la propria utilità, resta univocamente determinata la coppia di strategie in tal senso - razionali: $(2,2)$. Ma avviene il seguente paradosso: se entrambi $i$ giocatori si comportano in modo "irrazionale" (cioè scelgono la strategia 1 , "non confessare") ne traggono un vantaggio ${ }^{21}$.

La scelta è in genere considerata "razionale" quando presuppone un ragionamento volto a mettere in relazioni mezzi e fini, in vista di un vantaggio personale. Se i due prigionieri fossero però addestrati a non confessare mai, a mettere la causa per cui agiscono al di sopra dei propri interessi personali e avessero totale fiducia l'uno nell'altro (circostanza che talvolta accade quando i prigionieri sono membri di organizzazioni mafiose, gruppi terroristici o forze speciali), potrebbero fare la scelta più vantaggiosa per entrambi, anche senza possibilità di comunicare e pur non cercando il proprio tornaconto. In termini molto generali, possiamo notare che nei giochi a somma non nulla diventano "preponderanti gli aspetti psicologici, soggettivi, persino etici del problema, che rendono estremamente difficile una teorizzazione efficace» ${ }^{22}$. Il "dilemma del prigioniero" appartiene proprio a questo tipo di giochi.

Il successo di questo modello è dovuto al fatto che è stato elaborato in piena guerra fredda e spesso applicato per analizzare casi diplomatico-militari, come la crisi dei missili cubani o la corsa agli armamenti.

Nel 1951, Nash contribuisce in modo fondamentale allo sviluppo della teoria dei giochi con la pubblicazione di un articolo intitolato Non-Cooperative Games. Il lavoro riguarda il cosiddetto "Equilibrio di Nash". Osborne e Rubinstein definiscono l'equilibrio di Nash come un concetto di soluzione di un gioco non cooperativo "che coinvolge due o più giocatori, nel quale si presume che ogni giocatore conosca la strategia di equilibrio degli altri giocatori e nessun giocatore ha qualcosa da guadagnare nel cambiare soltanto e unilateralmente la propria strategia» ${ }^{23}$.

Nash definisce l'equilibrio di una strategia mista per ogni gioco con un insieme di azioni finito. Inoltre, dimostra che un equilibrio di Nash deve esistere in questo tipo di gioco. Per questo contributo, com'è noto, al matematico verrà attribuito il premio Nobel per l'economia nel 1994. Nash diventerà particolarmente famoso anche grazie al film A Beautiful Mind di Ron Howard (2001), che trae ispirazione da una versione romanzata della sua inusuale biografia, segnata da una grave malattia mentale.

21 Fürst: Teoria dei giochi.

22 Ibid.

23 Osborne/Rubinstein: A course in Game Theory. 
Nel 1957, Duncan Luce e Howard Raiffa, entrambi docenti della Columbia University, pubblicano un libro che avrà un ruolo molto significativo nel favorire il matrimonio tra teoria dei giochi e scienze sociali: Games and Decisions ${ }^{24}$. Molti scienziati sociali si trovavano infatti in difficoltà, per carenza di competenze, nel maneggiare i modelli elaborati dai matematici. Luce e Raiffa producono un'introduzione non tecnica alla teoria dei giochi e delle discipline correlate, alla portata dei non specialisti, che ha anche il dono di presentare tutti i casi più popolari: giochi a somma zero con due agenti, giochi non a somma zero con due agenti, giochi con n-agenti, processo decisionale individuale e di gruppo. Il libro viene scritto sotto gli auspici di Paul Lazarsfeld che, sempre alla Columbia University, era all'epoca impegnato nel tentativo di matematizzare la sociologia. Nella stessa università, con Lazarsfeld lavorava anche Merton, anch'egli interessato a dare alla sociologia un fondamento scientifico e teoretico solido. Tuttavia, come ci ricorda Swedberg, nessuno dei due sociologi adotterà i modelli matematici della teoria delle decisioni interattive ${ }^{25}$.

Nello stesso periodo, una certa attenzione alle possibili sinergie tra teoria sociologica e teoria delle decisioni interattive viene prestata dal sociologo francese Michel Crozier. Nel 1964, Crozier pubblica infatti The Bureaucratic Phenomenon ${ }^{26}$ - un classico della letteratura sociologica - ove viene fatto uso della game theory per comprendere certi fenomeni che avvengono nell'ambito delle strutture burocratiche. Dopo Crozier, a contribuire alla penetrazione nella sociologia francese dell'individualismo metodologico, della teoria delle decisioni razionali e della teoria dei giochi sarà soprattutto Raymond Boudon ${ }^{27}$.

Nel 1970, per la prima volta, un teorico dei giochi vince il Premio Nobel: è l'economista Paul Samuelson. Due anni più tardi, vincerà il Nobel Kenneth Arrow, un altro economista coinvolto nella game theory. Entrambi gli studiosi si distinguono per contributi allo sviluppo di una teoria dell'equilibrio in economia. Nel complesso, saranno 12 gli specialisti di teoria dei giochi a vincere il Premio Nobel. Questi straordinari risultati attraggono molti altri scienziati, attivi in diversi settori disciplinari, verso questo campo di studi.

\section{L'UltimATUM GAME}

Tra i giochi che favoriscono la sinergia tra diverse prospettive disciplinari, spicca in particolare l'ultimatum game. Si tratta di un modello matematico destinato a

24 Luce/Raiffa: Games and Decisions.

25 Swedberg: Sociology and Game 'Theory: Contemporary and Historical Perspectives.

26 Crozier: The Bureaucratic Phenomenon.

27 Boudon: Il posto del disordine. 
grande successo, elaborato nel 1982 da Werner Güth, Rolf Schmittberger e Bernd Schwarze. Lidea di base è molto semplice: due giocatori interagiscono per decidere come dividere una somma di denaro, sulla base di certe regole.

La rappresentazione in forma estensiva di un ultimatum game a due proposte è la seguente: il giocatore 1 può formulare una proposta equa o fair $(F)$ oppure iniqua o unfair $(\mathrm{U})$; il giocatore 2 può accettare $(A)$ o rifiutare $(\mathrm{R})$.

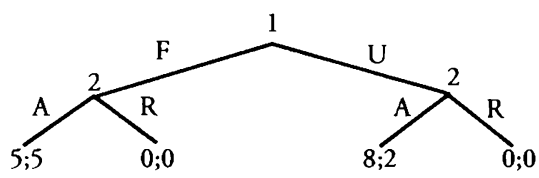

Le regole base sono le seguenti:

1) i soldi sono affidati a un solo giocatore;

2) questi formula l'offerta, proponendo come dividere la somma;

3) il secondo giocatore può accettare o rigettare la proposta;

4) se il secondo giocatore accetta, i soldi sono divisi secondo la proposta;

5) se questi rifiuta, entrambi i giocatori rimangono a mani vuote.

Questo è un gioco non cooperativo, perché i giocatori prendono le decisioni in modo indipendente. Ciò significa che i giocatori sono posti in competizione tra loro e non possono negoziare. Ciononostante, al fine di ottenere un risultato soddisfacente, una cooperazione tacita è necessaria. La cooperazione deve essere autoimposta (self-enforcing). Il fattore "reciprocità" non entra in gioco, perché l'ultimatum game è giocato una sola volta (one-shot game).

Una rappresentazione semi-formale del gioco è la seguente. La quota totale dei soldi disponibili è $\mathrm{x}$. Il primo giocatore sceglie una somma da proporre $\mathrm{p}$ nell'intervallo $[0, x]$. Il secondo giocatore sceglie una funzione $f:[0, x] \rightarrow$ \{"accetta", "rifiuta" $\}$. Possiamo rappresentare il profilo della strategia come $(p, f)$, dove $p$ è la proposta e $f$ è la funzione. Se $f(p)=$ "accetta", il primo riceve $p$ e il secondo $x-p$, altrimenti entrambi ottengono zero. Se $f(p)=$ "accetta" e non c'è $y>p$ tale che $f(y)=$ "accetta", allora $(p, f)$ è un equilibrio di Nash (NE) dell'ultimatum game. In parole semplici, l'equilibrio di Nash si raggiunge quando una proposta $p$ è formulata, tale che il giocatore 2 rigetterebbe tutte le proposte nelle quali il giocatore 1 ricevesse più di $p$.

Il risultato di questo esperimento è stato presentato in un articolo intitolato "An Experimental Analysis of Ultimatum Bargaining", pubblicato sul Journal of Economic Behavior and Organization ${ }^{28}$. L'aspetto più curioso dell'ultimatum game è che i risultati attesi, sulla base della teoria economica, non coincidono con i risultati empirici. L'economia neoclassica è notoriamente basata sull'idea di homo 
economicus - un agente razionale che cerca di massimizzare l'utilità come consumatore e il profitto come produttore. L'idea può essere fatta risalire a John Stuart Mill. Il filosofo inglese afferma infatti che, analogamente alla geometria, che presuppone un'arbitraria definizione di linea, come un ente che ha lunghezza ma non larghezza, «'economia politica presuppone un'arbitraria definizione di uomo, come un essere che inevitabilmente fa ciò che gli consente di ottenere la più grande quantità di beni necessari, convenienti e di lusso, con la più piccola quantità di lavoro e autonegazione fisica con la quale possono essere ottenuti» ${ }^{29}$.

Secondo J. S. Mill, un agente razionale può essere definito come "colui che è capace di giudicare l'efficacia comparativa dei mezzi necessari per ottenere quei fini» ${ }^{30}$. Questa definizione rappresenta la pietra angolare della teoria delle decisioni razionali o "rational choice theory".

Se definiamo l'utilità nei termini più ristretti di "guadagno monetario" (o profitto materiale) segue che l'affermazione "gli esseri umani tendono a massimizzare le utilità" diventa testabile o verificabile empiricamente. L'ultimatum game è un buon test per la teoria dell'homo economicus, perché anticipa chiaramente un risultato: il giocatore 2 accetterà qualsiasi offerta del giocatore 1 , quando $p>0$.

Si noti che lo stesso Mill mostra consapevolezza del fatto che la sua definizione di uomo è "arbitraria". Si tratta di una semplificazione che riesce a rappresentare piuttosto bene l'uomo borghese occidentale, protagonista della rivoluzione industriale e capitalista del XIX secolo. Restringere le utilità ai soldi è una forzatura ancora maggiore, ma questo è proprio quello che tendono a fare, più o meno consapevolmente, gli economisti (neo)classici.

I risultati empirici vanno però in tutt'altra direzione. Innanzitutto, non molti giocatori 1 offrono piccole quantità di denaro al giocatore 2 . Inoltre, quanto queste piccole quote sono proposte, le offerte vengono sistematicamente rifiutate. Questo quadro sembra avere validità quasi universale. Perlomeno, nei paesi industrializzati, i giocatori tendono a proporre una divisione più o meno "equa" della somma (es., $50: 50$ ), e tutte le offerte minori del $20 \%$ sono di norma rifiutate ${ }^{31}$.

Che cosa significa tutto ciò? I risultati possono essere interpretati prima facie come una confutazione della teoria dell'homo economicus. Sembra che gli esseri umani non siano interessati soltanto alla massimizzazione delle utilità (materiali, monetarie), ma anche a rinforzare gli standard etici della comunità - dove l'etica è qui intesa come senso di equità.

Da un punto di vista sociologico, l'ultimatum game è particolarmente importante, perché illustra la riluttanza umana all'accettazione delle ingiustizie. La tendenza a rifiutare piccole somme di denaro, a fronte di quote consistenti trattenute dal giocatore 1, può anche essere vista come rilevante per la definizione del concetto

29 Mill: On the Definition of Political Economy, and on the Method of Investigation Proper to It, p. 101.

30 Ibid.: p. 97.

31 Henrich [et al.]: Foundations of Human Sociality. 
di onore. Anche la teoria marxista della rivoluzione sociale - basta su concetti come "stratificazione sociale", "lotta di classe", "coalizione", "ribellione", "plusvalore", "pluslavoro", ecc. - può essere compresa nei termini della teoria dei giochi e in particolare dell'ultimatum game. La lotta di classe, che può prendere la forma di scioperi o di ribellioni armate - e che può comportare per i lavoratori anche la perdita del lavoro, della libertà o della vita - è generata dalla presa di coscienza dell'iniquità della proposta di suddivisione dei guadagni aziendali in profitti ingenti per i capitalisti (giocatore 1) e salari miseri per i lavoratori (giocatore 2).

In termini molto generali, se interpretiamo l'ultimatum game da un punto di vista biosociologico o evoluzionistico, possiamo notare che il mondo sociale e naturale favorisce la sopravvivenza e la riproduzione di individui "moderatamente egoisti", mentre è ostile agli individui "estremamente egoisti".

\section{IL DIBATTITO SUI RISULTATI DELL'ULTIMATUM GAME}

Nel 1989, Jack Ochs e Alvin Roth pubblicano un articolo intitolato "An Experimental Study of Sequential Bargaining" ${ }^{2}$, nel quale viene proposta una diversa interpretazione dei risultati dell'ultimatum game. Gli autori sostengono che i risultati empirici non mettono affatto in crisi i postulati del paradigma della rational choice theory. In estrema sintesi, sostengono che gli individui stanno comunque massimizzando le utilità attese, soltanto che dette utilità non sono traducibili direttamente in denaro contante.

Ovviamente, molto dipende dalla nostra definizione di razionalità o utilità. Per esempio, se includiamo l'autostima o il senso di giustizia nelle nostre utilità, allora il comportamento esibito negli esperimenti dell'ultimatum game è pienamente razionale. I giocatori subiscono danni psicologici quando accettano le offerte di piccole somme, mentre ottengono benefici psicologici quando le rifiutano, infliggendo una punizione al giocatore egoista.

Non è la prima volta che viene presentata questa interpretazione estesa del concetto di utilità, con un allargamento semantico ai benefici psicologici o spirituali e c'è senz'altro un grano di verità in questa ridefinizione. Tuttavia, il filosofo argentino Mario Bunge ha giustamente notato che l'asserto teorico «l'uomo d'affari razionale cerca sempre di massimizzare i guadagni» è solo una tautologia se il termine "razionale" è definito come "massimizzatore di utilità", ove tra l'altro l'utilità è definita in modo così esteso da includere qualsiasi cosa l'attore desideri e intenda ottenere. Il test empirico di una predizione, ovvero di una asserzione ex ante, si può effettuare soltanto se si mettono i termini dell'asserzione in relazione a possibili 
stati di realtà limitati e ben precisati. Per dirla in termini popperiani, la teoria è davvero scientifica soltanto se offre il collo alla mannaia. L'estensione del concetto di utilità sembra invece un'ipotesi ad hoc, che salva il paradigma economicistico, ma solo al prezzo di trasformarlo in una tautologia.

È però possibile un'altra interpretazione capace di salvare sia la teoria delle utilità che i fenomeni (i risultati dell'ultimatum game). Rifiutare le offerte di piccola entità può anche essere visto come un investimento in vista di futuri guadagni monetari o materiali. Avendo il potere di rifiutare l'offerta, il giocatore 2 utilizza detto potere come leva contro il giocatore 1 , al fine di motivarlo ad essere più equo in futuro. Punire gli individui troppo egoisti rende l'ambiente sociale più sicuro per tutti, incluso il giocatore 2 , che vive in quell'ambiente e potrà in futuro trarre beneficio economico dalla diffusione del "meme" dell'equità e della giustizia. Non ci sono però evidenze empiriche sufficienti per suggerire che questo calcolo razionale abbia davvero luogo nella mente del giocatore 2 . Al contrario, come vedremo tra poco, pare che sia una reazione emotiva, più che un calcolo razionale, a determinare il risultato.

\section{LA SINERGIA CON LE NEUROSCIENZE}

Nel 1994, John Harsany, John Nash e Reinhard Selten vincono il Premio Nobel per l'economia. Non è la prima volta che un teorico dei giochi ottiene l'ambito riconoscimento, ma è la prima volta che l'espressione "game theory" è menzionata esplicitamente nelle motivazioni. I tre studiosi sono infatti premiati "per la loro pionieristica analisi degli equilibri nella teoria dei giochi non-cooperativi» ${ }^{33}$.

Questo riconoscimento è un punto di svolta, perché non solo motiva più matematici e scienziati sociali ad immergersi nella teoria delle decisioni interattive, ma aiuta anche a reclutare alla causa scienziati naturali, specialmente biologi e neuroscienziati.

I frutti non tardano a venire. Nel 2003, Alan Sanfey e il suo team di ricerca (J. K. Rilling, J. A. Aronson, L. E. Nystrom, J. D. Cohen) pubblicano un articolo riguardante l'ultimatum game sulla prestigiosa rivista Science. Lo scritto è intitolato: "The neural basis of economic decision-making in the ultimatum game" ${ }^{34}$.

I ricercatori spiegano che

i modelli economici standard delle procedure decisionali umane (come la teoria delle utilità) hanno tipicamente minimizzato l'influenza delle emozioni sul

33 <http://www.nobelprize.org/nobel_prizes/economics/laureates/1994/> [12.01.2013].

34 Sanfey/Rilling/Aronson/Nystrom/Cohen: The neural basis of economic decision-making in the ultimatum game. 
comportamento decisionale delle persone, idealizzando il decisore come una macchina cognitiva perfettamente razionale. Comunque, negli ultimi anni questo assunto è stato sfidato dagli economisti comportamentali, i quali hanno identificato fattori psicologici ed emozionali addizionali che influenzano il processo decisionale ${ }^{35}$.

Da qualche anno a questa parte, i ricercatori hanno anche iniziato ad utilizzare le tecnologie di neuro-immagine per esaminare il comportamento dei decisori nei giochi economici. Il team scientifico guidato da Sanfey si è infatti avvalso della risonanza magnetica funzionale ( $\mathrm{fMRI}$ - functional magnetic resonance imaging), per scannerizzare 19 partecipanti impegnati a giocare l'ultimatum game nel ruolo di rispondente (giocatore 2).

Nell'esperimento, le offerte sono state formulate da giocatori reali e da computer. Le offerte provenienti dagli esseri umani seguivano comunque un algoritmo predeterminato, al fine di esporre tutti i partecipanti ad una gamma completa di offerte ed esattamente allo stesso insieme di possibilità. Metà di queste offerte erano eque, ovvero, proposte di dividere in parti eguali i 10 dollari a disposizione (\$5:\$5). Le rimanenti proposte erano invece basate su inique ripartizioni (due offerte di $\$ 9: \$ 1$, due offerte di $\$ 8: \$ 2$, e una offerta di $\$ 7: \$ 3$ ). Le 10 offerte provenienti dal giocatore computer erano identiche a quelle formulate dai partner umani (metà eque, metà inique).

I risultati comportamentali registrati sono simili a quelli usualmente rilevati negli esperimenti dell'ultimatum game. I partecipanti hanno accettato tutte le proposte eque, con un grado di accettazione decrescente a mano a mano che le offerte divenivano meno eque.

$\grave{E}$ interessante il fatto che le offerte inique di 1 e 2 dollari formulate da giocatori umani sono state rifiutate a un tasso significativamente superiore di quelle provenienti da un computer. Il che suggerisce che i partecipanti hanno una reazione emozionale più intensa alle proposte inique provenienti da esseri umani, piuttosto che da macchine.

La vera novità dell'esperimento di Sanfey è comunque nell'uso della risonanza magnetica funzionale. Le aree del cervello che mostrano una maggiore attività in presenza di offerte inique provenienti da esseri umani, se comparate a quanto accade in presenza di offerte eque, sono le seguenti tre: 1) insula anteriore bilaterale; 2) corteccia prefrontale dorsolaterale (CPD); 3) corteccia cingolata anteriore (CCA).

L'attivazione dell'insula bilaterale anteriore, in presenza di offerte inique provenienti da giocatori umani, è particolarmente interessante per via della notoria associazione di questa regione con gli stati emozionali negativi. Lattivazione dell'insula anteriore è stata infatti osservata invariabilmente negli studi del dolore 
e dell'angoscia, della fame e della sete, e dell'eccitazione autonomica, effettuati con le tecnologie della neuroimmagine. Sanfey e il suo team si esprimono in questi termini:

Tra questi stati emozionali negativi predominano la rabbia e il disgusto, i quali sono stati trovati innestati in distinte regioni dell'insula anteriore, attivate da una offerta iniqua nel presente studio. Sebbene gli studi del disgusto si sono incentrati maggiormente sulle sensazioni fisiche del gusto e dell'olfatto, è stato suggerito che il disgusto bastato sulle emozioni (come forse indotto da una offerta iniqua al limite dell'insulto) può essere concettualmente simile. L'attivazione di strutture neurali simili, precisamente l'insula anteriore, sia nel caso del disgusto fisico che nel caso del disgusto morale, fornisce qualche supporto a questa nozione ${ }^{36}$.

Sebbene sia ancora dibattuta l'idea stessa che si possa associare strettamente un'area del cervello con una precisa funzione, è comunemente accettata la tesi che la corteccia prefrontale dorsolaterale (CPD) sia associata a processi cognitivi come la gestione degli obiettivi e il controllo esecutivo. Perciò, i ricercatori della Princeton University hanno ipotizzato che l'attivazione della CPD che hanno osservato in risposta alle offerte inique possa essere correlata alla rappresentazione e al mantenimento della richiesta cognitiva del compito assegnato: ovvero, all'obiettivo di accumulare la maggior quantità possibile di denaro. Infatti, «'attività della CPD rimane relativamente costante, mentre l'insula anteriore si relaziona monotonicamente al grado di iniquità, riflettendo la risposta emozionale all'offerta ${ }^{37}$.

Infine, è stato notato da Sanfey che le offerte inique erano anche associate con un'attività accresciuta della corteccia cingolata anteriore. La CCA è implicata nel rilevamento di conflitti cognitivi, pertanto «l'attivazione qui potrebbe riflettere il conflitto tra motivazioni cognitive ed emozionali nell'Ultimatum Game» ${ }^{38}$.

In estrema sintesi (e in parole semplici), la parte razionale della nostra mente individua una possibilità di guadagno e si attiva in accordo, tuttavia, di fronte all'estremo egoismo di un essere umano, si attiva anche la parte del cervello predisposta a gestire forti sensazioni di disgusto, analoghe a quelle che proviamo venendo a contatto con sostanze maleodoranti o particolarmente nauseabonde. Il conflitto tra le due attività cerebrali attiva un "arbitro", il quale però - nella maggior parte dei casi - decreta la vittoria delle emozioni sul calcolo.

Tenuto conto che l'uomo è un animale sociale, risultato di milioni di anni di evoluzione, si può ipotizzare che si tratti di un meccanismo atto a garantire la coesione sociale. L'etica della coesione è un argomento affrontato anche da Jacques 
Monod nel suo libro più noto: Il caso e la necessita ${ }^{39}$. La coesione sociale è necessaria per la sopravvivenza del gruppo e degli individui. L'egoismo estremo di certi individui mette a rischio la coesione e attiva risposte volte a punire questi comportamenti o ad ostracizzare i soggetti pericolosi.

\section{II. RUOLO DELL'OXITOCINA NELL'ULTIMATUM GAME E NEL DICTATOR GAME}

Gli esperimenti dell'ultimatum game offrono un quadro di risposte tutto sommato coerente e consistente con la nostra conoscenza di sfondo biosociologica (pur permanendo un problema di compatibilità con i postulati dell'economia neoclassica). Tuttavia, se le risposte si strutturano secondo un modello riconoscibile, siamo pur sempre di fronte a regolarità statistiche e non a risposte omogenee ed unanimi. In altre parole, ci sono comunque esseri umani che accettano proposte inique o che mostrano sensibilità diverse nell'atto di formulare proposte tendenzialmente eque. Ora la domanda è: perché gli esseri umani si comportano diversamente? In che misura questo diverso comportamento dipende da fattori biologici, piuttosto che culturali?

La visione egualitaria dell'uomo, oggi dominante a livello politico, ci ricorda spesso e giustamente che gli esseri umani sono molto simili tra loro, e che pertanto sono ingiustificate le discriminazioni sulla base di specifiche caratteristiche individuali (come sesso, gruppo etnico, età, nazionalità, credo religioso, orientamento sessuale, ecc.). Tuttavia, questa visione politico-culturale non di rado ci fa scordare che "simili" non significa "uguali". Tutti i discorsi sulla "natura umana", pur legittimi, sono soltanto ipotetiche generalizzazioni ontologiche che mescolano dati empirici a posizioni ideologiche. Quantunque oggi non appaia politicamente corretto, un serio approccio scientifico non può mettere in ombra il fatto che la diversità tra gli esseri umani non attiene soltanto alla dimensione culturale, ma anche a quella fisiologica. Detta diversità può essere non rilevante sul piano dei diritti civili, ma ciò non significa che la scienza debba ignorarla. Uno studio della biodiversità umana sarebbe giustificato quand'anche fosse mosso da mera curiosità scientifica.

Sappiamo che le persone dimostrano diversi gradi di capacità cognitive, di empatia, di altruismo, di egoismo, di aggressività, di socialità, ecc. Sappiamo anche che queste caratteristiche dipendono in parte dall'ambiente (famigliare, scolastico, culturale, ecc.), e in parte da caratteristiche biofisiche. Nella misura in cui queste ultime sono coinvolte, diventa possibile intervenire in modo chimico-meccanico sulla dimensione emotivo-caratteriale, e dunque sulle decisioni e sui comporta- 
menti degli esseri umani, bypassando i consueti processi di socializzazione ed educazione.

In questa prospettiva di apertura a diverse ipotesi esplicative si muove la ricerca effettuata nel 2007, da Paul Zak, Angela Stanton e Sheila Ahmadi ${ }^{40}$. Detto studio mostra infatti che due fattori possono spiegare offerte generose nell'ultimatum game: la presa di prospettiva (perspective-taking) e l'empatia. Per esempio, i ricercatori hanno modificato la prospettiva dei partecipanti facendoli giocare l'ultimatum game in diversi ruoli, ovvero come giocatore 1 e giocatore 2 . Scambiandosi i ruoli i giocatori hanno acquisito una maggiore capacità di comprendere il partner di gioco e quindi di evitare il rifiuto dell'offerta, che come sappiamo comporta una perdita netta di profitto monetario per entrambi.

Gli autori discutono nell'articolo le diverse teorie dell'altruismo in circolazione. Distinguono anche l'altruismo dalla generosità, stabilendo che quest'ultima implica un dare più di quanto sia atteso o necessario. Discutono anche il ruolo dell'empatia nel generare atti di altruismo o generosità, richiamando gli studi di Tommaso d'Aquino, David Hume e Adam Smith.

L'aspetto però più interessante della ricerca è che vengono investigati empiricamente gli aspetti fisiologici della questione e, in particolare, il ruolo dell'empatia nel produrre generosità attraverso la manipolazione di un meccanismo fisiologico che si ipotizza istanziare l'empatia: il neuromodulatore ossitocina (OT). «Studi recenti sugli esseri umani hanno infatti dimostrato che l'OT facilità un temporaneo attaccamento tra sconosciuti, aumentando la fiducia e la reciprocità ${ }^{41}$.

Zak, Stanton e Amhadi cercano dunque ulteriori conferme del fatto che l'ossitocina è un meccanismo che aumenta la generosità tra esseri umani che non si conoscono. Per dissociare il ruolo fisiologico dell'empatia nel produrre generosità e altruismo sono stati assegnati ai partecipanti due compiti diversi, entrambi basati su un trasferimento di denaro finalizzato a garantire una misura oggettiva delle due attitudini. Così gli autori riassumono il modello matematico adottato:

Si consideri una interazione diadica tra due individui, $i$ e $j$. Sia $b_{i}$ il beneficio che $i$ riceve, e $b_{j}$ il beneficio di $j$. Gli individui ottengono utilità dal ricevere $\mathrm{i}$ propri benefici e possibilmente dall'altra persona che riceve benefici. Includiamo un parametro $\alpha €[0,1]$ che coglie l'empatia che una persona ha per l'altra. Utilizziamo il termine empatia nel suo significato consueto di "un'identificazione con e una comprensione di sentimenti o situazioni di un altro". Ci attendiamo che $\alpha$ sia più alto quando si è indotti a considerare esplicitamente $\mathrm{i}$ sentimenti del proprio partner diadico in relazione ai benefici che vengono offerti. Questo è stato chiamato "presa di prospettiva" dagli psicologi sociali. Per semplicità, sia l'utilità data da una forma standard $b^{\beta}$, dove $\beta \in(0,1)$. Siano i 
benefici totali limitati, $\mathrm{b}_{\mathrm{j}}+\mathrm{b}_{\mathrm{j}}=\mathrm{M}<\infty$. Se a una persona $i$ chiediamo di dividere i benefici $M$ tra se e la persona $j$, allora $i$ affronta il seguente problema di massimizzazione delle utilità,

$$
\begin{aligned}
& \operatorname{Max}_{b i b j} b_{i}^{\beta}+a b_{j}^{\beta} \\
& \text { s.t. } \quad b_{i}+b_{j}=M \\
& b_{j} \geq 0 .
\end{aligned}
$$

Quando $\alpha=0$, l'individuo $i$ è completamente egoista, quando $\alpha=l$ egli/ella è egualitario ${ }^{42}$.

Da queste premesse segue che il beneficio offerto dall'individuo $i$ all'individuo $j$ cresce quando $\alpha$ è alto. La strategia sperimentale consiste nell'indurre i partecipanti a prendere in considerazione la possibilità di essere puniti a causa di un'offerta iniqua, proprio come accade nell'ultimatum game, e di confrontare il comportamento in questo gioco con quello assunto in un gioco differente dove non è prevista punizione. Questo secondo gioco è il cosiddetto "dictator game", che non abbiamo ancora incontrato e discusso in dettaglio in questo scritto, ma può essere riassunto in poche parole: il giocatore 1 fa un'offerta e il giocatore 2 può solo accettarla passivamente. Poiché nel trasferimento unilaterale non è richiesto di considerare la prospettiva dell'altro, ci si aspetta che questo compito produca un $\alpha$ più basso $\mathrm{e}$ un corrispondente trasferimento monetario di minore entità. Ed ecco come i ricercatori espongono l'idea fondamentale dell'esperimento: «Al fine di dimostrare l'effetto causale dell'OT sulla generosità, abbiamo infuso metà dei partecipanti con OT per via intranasale mentre l'altra metà ha ricevuto la stessa quantità di soluzione salina normale» ${ }^{43}$. Precisamente, ai partecipanti viene somministrata per via intranasale una dose di $40 \mathrm{IU}$ di ossitocina (OT) o di placebo (soluzione salina).

I ricercatori definiscono un trasferimento generoso quello in cui il giocatore 1 offre una somma che eccede la media delle offerte minime accettabili nell'ultimatum game. In parole semplici, le offerte generose sono quelle più alte di quelle considerate accettabili.

Poiché nel dictator game non è prevista la possibilità di rifiutare l'offerta, c'è generale consenso tra gli economisti sperimentali sul fatto che il trasferimento monetario nell'ambito di questo gioco possa essere trattato come una misura dell'altruismo. Perciò, sottoporre lo stesso gruppo di partecipanti all'ultimatum game e al dictator game consente de facto di dissociare la generosità dall'altruismo.

I risultati dell'esperimento sono stati giudicati significativi, se non addirittura sorprendenti. La media delle offerte da parte del giocatore 1 nell'ultimatum game è risultata più alta del $21 \%$ nel gruppo a cui è stata somministrata l'ossitocina, 
rispetto al gruppo esposto al placebo: media OT $\$ 4.86\left(\mathrm{DS}^{44} \$ 1.06\right)$; media placebo $\$ 4.03$ (DS $\$ 1.29$ ). Per quanto riguarda la media dell'offerta minima accettabile, è stato scoperto che la generosità era più alta dell' $80 \%$ nei gruppi OT rispetto ai gruppi placebo. La media del gruppo OT è infatti risultata di \$1.89 (DS \$1.06); mentre la media del gruppo placebo ammonta a \$1.06 (DS \$1.29).

L'inalazione di ossitocina provoca invece un cambiamento meno significativo delle risposte nel dictator game, dove si registra una media del gruppo OT di $\$ 3.77$ (DS \$2.21); e una del gruppo placebo di \$3.58 (DS \$2.15). Risulta tuttavia confermato che le offerte sono mediamente inferiori.

Sebbene i ricercatori abbiano aumentato artificialmente i livelli di ossitocina, al fine di stabilire un meccanismo causale capace di produrre generosità, viene anche sottolineato che il livello di OT può essere aumentato in modo non farmacologico, in una varietà di modi, tra i quali il contatto tattile, l'interazione in un ambiente sicuro, o lo scambio di segnali di fiducia da parte delle persone che interagiscono.

\section{Il RUOLO DEL TESTOSTERONE NELL'ULTIMATUM GAME}

Nel 2007, la conoscenza dei processi biologici che influenzano le decisioni economiche si è ampliata grazie ad una ricerca di Terence C. Burnham ${ }^{45}$, ricercatore dell'Università di Harvard. Burnham ha messo insieme un gruppo di 26 studenti di microeconomia e ha chiesto loro di giocare l'ultimatum game. In principio, $i$ partecipanti dovevano essere 30 , ma quattro di loro non si sono presentati all'esperimento. Tutti gli studenti reclutati erano di sesso maschile.

Per quanto riguarda il metodo, l'esperimento è stato disegnato in modo tale da indurre i giocatori a formulare un numero significativo di offerte inique, al fine di ottenere un numero apprezzabile di rifiuti. Le offerte basse sono in genere piuttosto rare, per i motivi che abbiamo già spiegato. Per evitare una preponderanza di offerte eque, si è quindi deciso di escludere la ripetizione del gioco. Il cambio di prospettiva e la reiterazione influenzano infatti i giocatori, inducendoli a convergere verso offerte eque. Inoltre, è stato stabilito che i 40 dollari affidati ad ogni coppia di giocatori, per l'eventuale spartizione, non potessero essere divisi a piacimento. Le opzioni di offerta sono infatti state limitate a sole due possibilità: 25 dollari o 5 dollari. In altre parole, il giocatore 1 , che aveva la disponibilità del denaro ed era chiamato a formulare l'offerta, non poteva dividerla in parti eguali. $\mathrm{O}$ si teneva 15 dollari, ossia meno della metà del totale, lasciando la quota maggiore al gioca- 
tore 2 , oppure cercava di portarsi a casa 35 dollari, lasciando le briciole al giocatore 2 .

Inoltre - e questo è l'aspetto più interessante dell'esperimento - il Dr. Burnham ha prelevato un campione di saliva da ogni partecipante, al fine di rilevare il livello di testosterone e di metterlo a confronto con le decisioni assunte in ogni round del gioco e con quello degli altri giocatori.

I risultati dell'esperimento hanno confermato l'ipotesi di partenza: gli individui con il livello di testosterone più alto erano quelli più propensi a rigettare le offerte inique. L'ipotesi di lavoro era basata sull'assunto che un'offerta iniqua viene in genere interpretata come una sfida. Una sfida all'orgoglio o alla dignità della persona. Gli alti livelli di testosterone sono notoriamente associati all'aggressività e alla tendenza ad assumere posizioni dominanti, negli animali come negli esseri umani. Burnham ricorda che gli scimpanzé di alto rango (Pan troglodytes) hanno un livello di testosterone più alto di quello degli individui di basso rango, e sono più aggressivi. «Similmente, i maschi dominanti dei gorilla selvaggi di montagna (Gorilla gorilla beringei) hanno un livello di testosterone più alto di quello dei maschi subordinati» ${ }^{46}$. Perciò, era lecito ipotizzare una maggiore propensione dei giocatori 2 con alto livello di testosterone ad infliggere punizioni ai giocatori 1 che tentavano egoisticamente di trattenere 35 dollari. In dettaglio, secondo le previsioni, l'esperimento ha mostrato che

gli uomini che hanno rifiutato le basse offerte dell'ultimatum game ( $5 \$$ su $40 \$$ ) hanno un livello di testosterone significativamente più alto di coloro che le hanno accettate $(p<0.01$, one-sided $t$-test). Per ogni soggetto, il livello di testosterone è stato stimato facendo la media di tre campioni prelevati in giorni differenti... Coloro che hanno rigettato l'offerta (6) hanno un livello medio di testosterone di $383 \mathrm{pmoll}^{-1}$ contro una media di $251 \mathrm{pmoll}^{-1}$ per quelli che hanno accettato $(20)^{47}$.

La seguente tabella riassume i risultati dell'esperimento:

\begin{tabular}{|c|c|c|c|}
\hline & $N$ & media testosterone $\left(\mathrm{pmoll}^{-1}\right)$ & s.e. \\
\hline Rifiuta $\$ 5 / \$ 40$ & 6 & 383 & 37 \\
\hline Accetta \$5/\$40 & 20 & 251 & 16 \\
\hline Totale & 26 & 281 & \\
\hline
\end{tabular}


I giocatori che hanno rifiutato le offerte inique avevano dunque un livello di testosterone più alto del $50 \%$ della media di quelli che le hanno accettate. Cinque dei sette maschi che avevano il livello di testosterone più alto hanno rifiutato l'offerta di 5 dollari, mentre solo un giocatore degli altri 19 ha preso la stessa decisione.

Gli economisti spesso considerano irrazionale questo tipo di risposta, giacché andare a casa con 5 dollari è pur sempre preferibile che andare a casa a mani vuote. In realtà, se si guarda la questione da un punto di vista biosociologico, questo comportamento ha una sua razionalità intrinseca. I soldi esistono da qualche migliaio di anni, mentre l'uomo esiste da qualche milione di anni. Dunque la "vera" misura del valore di un uomo non possono essere i soldi. Deve essere qualcos'altro. I soldi possono essere talvolta la misura del valore di questo "qualcos'altro", ma limitatamente ad una società modellata sul sistema dei valori capitalistico-borghesi che esiste da qualche centinaio di anni. Il qualcos'altro di cui parliamo è lo "status sociale", un concetto che include il rispetto da parte di altri individui e il rispetto che una persona ha di se stessa. I soldi, nel presente periodo storico, sono dunque da vedere come un mero strumento per acquisire il vero fine, che è lo status sociale. Se viene offerto un altro modo per acquisire status sociale, si può scegliere razionalmente di seguire quella via. Talvolta è sufficiente fare la faccia cattiva, digrignare i denti, gonfiare il petto, altre volte sono necessarie punizioni più dure. Anche nel nostro caso, la via per attivare nel "branco" il rispetto verso il maschio dominante è la punizione di chiunque osi lanciare una sfida. Se per raggiungere lo scopo è sufficiente privare di un bene lo sfidante, piuttosto che acquisirne uno per sé, i soldi diventano del tutto irrilevanti. Anzi, la capacità di rinunciare ad essi diventa un ulteriore strumento per mostrare la propria superiorità e mettere a nudo la piccolezza morale dell'egoista.

Sebbene i risultati confermino l'ipotesi di partenza, questo esperimento può ancora sorprendere per l'alto numero di giocatori che hanno accettato le offerte inique (20 su 26). Il numero è considerevole, se raffrontato ad altri esperimenti di questo tipo. Questo risultato può trovare varie spiegazioni. Una possibile spiegazione è che le "cavie" erano tutti studenti di microeconomia e dunque soggetti particolarmente motivati al guadagno (altrimenti avrebbero studiato belle arti o storia) e, oltretutto, indottrinati con la rational choice theory, secondo la quale un operatore economico razionale cerca sempre di massimizzare il profitto. Ma la tipologia dei partecipanti è soltanto una delle possibili ipotesi interpretative. Questo aspetto non è stato discusso in dettaglio da Burnham, ma l'autore ha correttamente precisato che «sarebbe utile replicare questo studio con più individui $\mathrm{e}$ soggetti pescati da varietà di popolazioni» ${ }^{48}$.

Un'altra spiegazione potrebbe essere che i partecipanti avevano perfettamente capito che il giocatore 1 non aveva scelta. Doveva decidere se danneggiare se stesso 
o il giocatore 2. Per cui, l'egoismo veniva più frequentemente perdonato, perché imposto dal meccanismo del gioco. Paradossalmente, un meccanismo creato per produrre più offerte inique, grazie alla consapevolezza dei giocatori, ha anche generato più accettazioni di dette offerte. Se è corretta questa seconda interpretazione, a maggior ragione sono significativi i dati sull'influenza del testosterone.

Si potrebbe infine notare che questo esperimento non fa che confermare il senso comune: meglio non sfidare maschi adulti con alto livello di testosterone. Detto livello può essere rilevato con precisione da un test di laboratorio sulla saliva, secondo la procedura elaborata da Peter Ellison ${ }^{49}$. Tuttavia, anche la meno precisa analisi basta sull'esperienza di vita - che ci induce coscientemente o incoscientemente a rilevare se l'uomo che abbiamo di fronte ha folta peluria, mascella squadrata, dito anulare più lungo dell'indice, ecc. - può dare qualche risultato nel determinare il grado di aggressività potenziale di un maschio adulto.

Si deve però notare che, quand'anche lo studio non facesse altro che confermare il senso comune, è comunque necessaria una conferma scientifica, giacché non possiamo sapere a priori quali assunti della conoscenza ordinaria sono fondati e quali meri pregiudizi. Per migliaia e forse milioni di anni gli esseri umani hanno creduto che il mondo fosse piatto e immobile o che le specie viventi fossero fisse. Questi sono solo due esempi in cui la verifica scientifica ha gettato completamente all'aria le certezze del senso comune.

\section{IL RUOLO DELLA SEROTONINA NELL'ULTIMATUM GAME}

Nel 2008, Molly Crockett, Luke Clark, Golnaz Tabibnia, Matthew D. Lieberman e Trevor W. Robbins hanno pubblicato un altro interessante articolo sulla rivista Science intitolato "Serotonin Modulates Behavioral Reactions to Unfairness" ${ }^{0}$. In questo caso, i ricercatori dell'Università di Cambridge hanno provato (o perlomeno corroborato) per via sperimentale la tesi che c'e una correlazione tra basso livello di serotonina e risposta negativa alle offerte inique. Le persone a cui è stato artificialmente abbassato il livello di serotonina (5-HT) hanno infatti rifiutato le proposte inique più spesso dei giocatori con un livello di serotonina normale. Tuttavia, detti soggetti non hanno rifiutato le proposte eque, né hanno mostrato cambiamenti nell'umore, nell'equità dei giudizi, nei processi basilari di ricompensa o nell'inibizione delle risposte. I risultati sembrano dunque suggerire che la seroto-

49 Ellison: Human salivary steroids: methodological considerations and applications in physical anthropology.

50 Crockett/Clark/Tabibnia/Lieberman/Robbins: Serotonin Modulates Behavioral Reactions to Unfairness. 
nina gioca un ruolo critico nella regolazione delle emozioni durante i processi di decisione sociale. La premessa da cui partono i ricercatori è la seguente:

Una delle prime regole sociali che impariamo da bambini è la regola d'oro: tratta gli altri come vorresti essere trattato. Sfortunatamente, i nostri pari non sempre meritano medaglie per il loro comportamento, sicché siamo tentati dalla vendetta. Resistere agli impulsi aggressivi può essere difficile, ma per affrontare la vita sociale in modo vincente talvolta è necessario auto-regolarsi di fronte alle ingiustizie percepite. La serotonina $(5-\mathrm{HT})$ ha un ruolo nei comportamenti sociali, inclusa l'aggressione impulsiva, ma il suo preciso coinvolgimento nel controllo degli impulsi è controverso. Poiché l'interazione sociale può evocare emozioni forti, è plausibile che 5 -HT moduli l'impulsività attraverso meccanismi di regolazione delle emozioni ${ }^{51}$.

L'esperimento effettuato dai ricercatori di Cambridge era basato sullo studio degli effetti della manipolazione del 5-H'T nell'ultimatum game. Precisamente, seguendo una procedura a doppio cieco con controllo placebo, i ricercatori hanno provocato in 20 volontari sani un esaurimento acuto del triptofano (ATD), ottenendo il concomitante abbassamento temporaneo dei livelli di serotonina ${ }^{52}$. Quindi hanno chiesto ai volontari di giocare all'ultimatum game, in diversi incontri singoli (oneshot game).

Le offerte potevano cadere in tre categorie di "equità": $45 \%$ della somma (equa), $30 \%$ della somma (iniqua), o $20 \%$ della somma (più iniqua).

I tassi di rigetto (\% di offerte rifiutate), è stato calcolato per ogni soggetto ad ogni livello di equità, durante i trattamenti ATD e placebo. Le analisi della varianza di misure ripetute hanno rivelato un'interazione fra trattamenti ed equità (fairness) altamente significativa $(F=6.891, P=0.003)$. Comparato al placebo, l'ATD ha significativamente incrementato il tasso di rigetto, e questo effetto è risultato limitato alle offerte inique. In contrasto, l'ATD non ha interagito in modo significativo con la magnitudine dell'offerta $(F=1.164, P=0.294)^{53}$.

Altre ricerche citate nell'articolo dimostrano che l'aumento del rigetto delle offerte inique, in seguito al trattamento ATD, non può essere facilmente ricondotto a cambiamenti dell'umore, dei giudizi di equità, o dei meccanismi di inibizione delle risposte. Lo studio apparso su Science ha ulterioriormente corroborato questa tesi.

51 Ibid.

52 È noto infatri che il triptofano è uno degli amminoacidi essenziali nella dieta degli esseri umani, mentre la serotonina è un composto organico azotato che deriva dal triptofano. Quest'ultimo deve essere assunto tramite l'alimentazione, giacché l'organismo umano non è in grado di sintetizzarlo.

53 Crockett/Clark/Tabibnia/Lieberman/Robbins: Serotonin Modulates Behavioral Reactions to Unfairness. 
In ogni sessione, i ricercatori hanno infatti "chiesto ai partecipanti di indicare la magnitudine di un'offerta equa per ogni somma, e l'ATD non ha influenzato questi giudizi $(F=0.648, P=0.431)$ ».

Come nel caso precedente, si potrebbe pensare che i ricercatori abbiano semplicemente trovato la conferma empirica rigorosa di una nozione del senso comune. Non è un caso se vengono organizzati "pranzi d'affari" e se a organizzarli è di norma il soggetto che ha un'offerta economica da fare (giocatore 1). È altresì risaputo che il partner economico (giocatore 2) è più malleabile a stomaco pieno che a stomaco vuoto. E questo vale anche per altri tipi di offerta, come vuole la norma sociale di far coincidere l'incontro galante con una "cena romantica". Tuttavia, questo esperimento ci insegna qualcosa di più preciso. Non basta un pasto qualsiasi per alterare il livello di triptofano, in un senso o nell'altro, giacché questo amminoacido è contenuto in misura diversa negli alimenti. Inoltre, il senso comune può dare anche indicazioni sconvenienti. Capita infatti che nei pranzi d'affari e nelle cene galanti, a seconda delle culture, vengano offerti alcolici, per la loro nota capacità di rendere malleabili o arrendevoli le persone. Ma questa offerta potrebbe rivelarsi un errore di gioco, proprio perché gli effetti dell'alcol sono noti a tutti. Il giocatore 2 potrebbe irrigidirsi, sospettando le intenzioni del giocatore 1. Difficilmente, però, la mossa di mettere in tavola agnello, sardine, formaggi, uova e altri alimenti ricchi di triptofano attiverebbe un analogo sospetto.

Possiamo dunque concludere che questi studi interdisciplinari non si limitano a confermare il senso comune, ma accrescono di un grado apprezzabile la nostra conoscenza delle dinamiche interattive e la possibilità di elaborare strategie vincenti.

\section{BIBLIOGRAFIA}

[Anonymus]: Émile Borel. In: Encyclopaedia Britannica. <http://www.britannica.com/EBchecked/topic/ 74060/Emile-Borel> [29.10.2013].

Aumann, R. J.: Game theory. In: Durlauf, S. N. (ed.): The New Palgrave Dictionary of Economics, Basingstoke ${ }^{2} 2008$ (1987).

Borel, É.: Algèbre et calcul des probabilités, Comptes Rendus Academie des Sciences 184, (1927).

Boudon, R.: Il posto del disordine. Critica delle teorie del mutamento sociale, Bologna 1984.

Burnham, T. C.: High-testosterone men reject low ultimatum game offers. In: Proceeding of the Royal Society $B$ (Biological Sciences), 2007 September 22; 274(1623), pp. 2327-2330.

Crockett, M./Clark, L./Tabibnia, G./Lieberman, M. D./Robbins, T. W.: Serotonin Modulates Behavioral Reactions to Unfairness. In: Science, 2008 June 27; 320(5884), p. 1739.

Crozier, M.: The Bureaucratic Phenomenon, Chicago 1964.

Dixit, A./Nalebuff, B.: Game Theory. In: The Concise Encyclopedia of Economics, Library of Economics and Liberty, 2008. <http://www.econlib.org/library/Enc/GameTheory.html> [29.10.2013].

Ellison, P. T.: Human salivary steroids: methodological considerations and applications in physical anthropology. In: Yearbook of Physical Anthropology 31, 1988, pp. 115-142. 
Fürst, D.: Teoria dei giochi. In: Enciclopedia delle scienze sociali, Treccani, 1994. <http://www.treccani.it/enciclopedia/teoria-dei-giochi_(Enciclopedia-delle-Scienze-Sociali)/> [29.10.2013].

Geerc, C.: Local Knowledge. Further Essays in Interpretive Anthropology, New York ${ }^{3} 2000$ (1983).

Güth, W./Schmittberger, R./Schwarze, B.: An Experimental Analysis of Ultimatum Bargaining. In: Journal of Economic Behavior and Organization 3:4 December 1982, p. 367-388.

Henrich, J. [et al.]: Foundations of Human Sociality, Oxford 2004.

Hossain, E./Niyato, D./Han, Z.: Dynamic Spectrum Access and Management in Cognitive Radio Networks, Cambridge 2009.

Kowalewicz, M. H.: Spieltheorie von Roger Caillos oder Spiel als Totalphänomen. In: Spiel. Facetten seiner Ideengeschichte, a cura di: id., Münster 2013, pp. 111-131.

Luce, D./Raiffa, H.: Games and Decisions, New York 1957.

Merton, R. K.: The Sociology of Science. Theoretical and Empirical Investigations, Chicago 1973.

Mill J. S.: On the Definition of Political Economy, and on the Method of Investigation Proper to It. In: Essays on Some Unsettled Questions of Political Economy, Kitchener ${ }^{2} 2000$ (1844).

Monod, J.: Il caso e la necessità. Saggio sulla filosofia naturale della biologia contemporanea, Milano 1971.

Morgenstern, O.: Game Theory. In: Dictionary of the History of Ideas. Studies of Selected Pivotal Ideas, Vol. II, ed. P. P. Wiener, New York 1973 (1968).

Myerson, R. B.: Game Theory: Analysis of Conflict, Harvard 1991.

Nash, J.: La teoria dei giochi. <http://www.youtube.com/watch?v=SrkCL7QrKkg> [29.10.2013].

Neumann, J. v.: Zur Theorie der Gesellschaftsspiele. In: Math. Annalen 100 (1928), pp. 295-320.

Neumann, J. v./Morgenstern, O.: Theory of Games and Economic Behavior, Princeton 1944.

Osborne, M./Rubinstein, A.: A course in Game Theory, Cambridge, Massachusetts 1994.

Poundstone, W.: Prisoner's Dilemma, New York 1992.

Ross, D.: Game Theory. In: The Stanford Encyclopedia of Philosophy (Winter 2012 Edition), a cura di: E. N. Zalta, <http://plato.stanford.edu/archives/win2012/entries/game-theory/> [29.10.2013].

Roth, A.: An Experimental Study of Sequential Bargaining. In: American Economic Review 79, pp. 355384.

Sanfey, A./Rilling, J. K./Aronson, J. A./Nystrom, L. E./Cohen, J. D.: The neural basis of economic decision-making in the ultimatum game. In: Science 300, pp. 1755 1758.

Schwalbe, U./Walker, P. : Zermelo and the Early History of Game Theory. In: Games and Economic Behavior, 34-1, 2001, pp. 123-137. <http:/www.math.harvard.-edu/-elkies/FS23j.03/zermelo.pdf> [29.10.2013].

Swedberg, R.: Sociology and Game Theory: Contemporary and Historical Perspectives. In: Theory and Society 30, n. 3, June 2001, pp. 301-335.

Walker, P.: A Chronology of Game Theory, September 2012. <http://www.econ.canterbury.ac.nz/personal_pages/paul_walker/gt/hist.htm> [29.10.2013].

Zak, P. J./Stanton, A. A./Ahmadi, S.: Oxytocin Increases Generosity in Humans. In: PLoS ONE 2(11), 2007: e1128.

Załuski, W.: Game Theory in Jurisprudence, Kraków 2013.

Zermelo, E.: Über eine Anwendung der Mengenlehre auf die Theorie des Schachspiels. In: Proceedings of the Fifth International Congress of Mathematicians, Vol. II, a cura di: E. W. Hobson/A. E. H. Love, Cambridge 1913, pp. 501-504. 